\title{
A retrospective analysis of Acute Lymphoblastic Leukemia incidence among hospitalized patients from Jan- 2017 to Sep-2021
}

Sajid Ali ${ }^{1}$, Ayesha Sadia ${ }^{2}$, Abid Jameel ${ }^{1}$, Sufyan ${ }^{1}$, Shah Hussain ${ }^{1}$ and Shehryar Ahmad ${ }^{3 *}$

1. Hayatabad Medical Complex Peshawar, Pakistan

2. Hematology KMU Peshawar, Pakistan

3. Health Department Khyber-Pakhtunkhwa, Pakistan

*Corresponding author's email: shery9444@gmail.com

Citation

Sajid Ali, Ayesha Sadia, Abid Jameel, Sufyan, Shah Hussain and Shehryar Ahmad. A retrospective analysis of Acute Lymphoblastic Leukemia incidence among hospitalized patients from Jan-2017 to Sep-2021. Pure and Applied Biology. Vol. 11, Issue 4, pp902-906. http://dx.doi.org/10.19045/bspab.2022.110092

\begin{tabular}{llll}
\hline \hline Received: 07/01/2022 & Revised: 25/02/2022 & Accepted: 03/03/2022 & Online First: 05/03/2022 \\
\hline
\end{tabular}

\section{Abstract}

Acute Lymphoblastic Leukemia (ALL) is heterogeneous group of genetically linked lymphoid neoplasm associated with increased incidence rate. This study was designed to determine the incidence of ALL in Khyber Pakhtunkhwa based on the age, gender and geographic distribution. A retrospective study was conducted by reviewing hospital record at Medical Oncology ward of Hayatabad Medical Complex Peshawar, Pakistan. A total of 161 patients were selected from January 2017 to September 2021 who met the criteria of ALL were included. The incidence was higher in children under ages under 23 years whereas the highest number of cases was reported from district DIR of Khyber Pakhtunkhwa province which is quite alarming. Male to female ratio was 1.27:1 respectively whereas male shows slightly predominance as compare to female. This study concludes increase incidence of ALL in children whereas increase frequency in District DIR population along with male predominance attract attention regarding further study to evaluate the possible etiologies behind increase incidence.

Keywords: Acute Lymphoblastic Leukemia; Bone Marrow; Flow cytometry; Khyber Pakhtunkhwa; Philadelphia

\section{Introduction}

Acute Leukemia is defined by the failure of early premature cells to differentiate into more mature cells as well as their uncontrolled growth leading to its clinical manifestations. Acute leukemia is mainly of two types i.e. acute lymphoblastic leukemia (ALL) and acute myeloblastic leukemia
(AML). Acute lymphoblastic leukemia is the arrest of lymphoid lineage at the blast level i.e. lymphoblast's in the bone marrow along with its aberrant and uncontrolled proliferation leading to the suppression of normal hematopoiesis [1]. This result in its manifestations of generalized weakness, fatigue, dyspnea, fever, infection, bleeding, 
hepatosplenomegaly, lymphadenopathy, tumor lysis syndrome in high TLC counts, SVC obstruction in case of mediastinal mass and body aches especially in children [1]. ALL can be either B or T lymphoid lineage, with B being about $80 \%$, T cell about $15 \%$ and Mature Burkitt leukemia about 5\% [2]. ALL can be either Philadelphia positive or negative [3].

A person of any age can develop ALL in any stage of life but higher incidence is found in children. Almost majority juvenile malignancies are caused by unknown factors. Potentiality in particular ionizing and nonionizing radiation are examples of environmental causal agents environmental pollutants, for example, play a little role in childhood leukemia. The incidence of ALL in the United States is estimated to be 1.6 per 100,000 people. 1 In 2016, an estimated 6590 new cases of ALL were detected, with over 1400 fatalities as a result of the disease (American Cancer Society). ALL has a bimodal incidence pattern, with the first peak occurring in childhood and the second peak happening around the age of 50.2, while dose intensification techniques have improved results for pediatric patients, the prognosis for the elderly is still quite dismal.

Risk of ALL is higher in males. Higher incidence in children under age 5 years with decreased mortality. The Risk increases after mid 50 years and peaks at old age with higher mortality due to poor cytogenetics and comorbidities [1-4]. Due to lack of cancer registry in Pakistan and especially in KPK there is no solid data available about the incidence of ALL in this population. The present study was aimed to provide a platform for the incidence of ALL in this corner of the world and will be a foundation for future studies to be performed. A true picture of acute lymphoblastic leukemia incidence based on age, gender will be provided and also the districts with most cases reported will also be a roadmap for further study.

\section{Materials and Methods}

A retrospective study was conducted at Medical Oncology ward Hayatabad Medical Complex in Peshawar, with 161 ALL patients selected from hospital data records between January 2017 and September 2021. Patients participated in the study varied in age from 5 to 90 years old and were of either gender who satisfied the criteria for ALL, which included at least $20 \%$ blasts in the bone marrow and supported confirmatory testing via cytochemistry, flow cytometry, or immunohistochemistry. Data regarding sociodemographic variables and other clinical variables were recorded on predesigned Proforma. Data were analyzed using Microsoft Excel and displayed in the form of tables and graphs. Participants were divided in to 08 different group based on age showing percentage wise distribution.

\section{Results}

In this study, the mean age of participant was 34 years. The participant was further divided into 08 different age groups which revealed 24 patients in age group $<10$ years, 22 in 10 20 years, 22 in $21-30$ years, 22 in $31-40$ years, 21 in 41- 50 years, 18 in 51-60 years, 15 in $61-70,10$ in 71-80 years while 7 patients were in age group $>80$ years respectively as shown in (Table $1 \&$ Fig. 1).

Regional wise analyses revealed the most frequent cases were from district DIR having frequency of 31 patients followed by Bajawar 28, Peshawar 22, Bannu 22, Mardan 15, kurram agency 06, Malakand 06, Swabi, Nowshehra, Dera Ismail Khan 05 each, Chitral, swat, karak 3 each, Buner and Mohmand agency 02, while least frequent 01 each from hangu, shangla, kohat respectively as shown in (Fig. 2). 


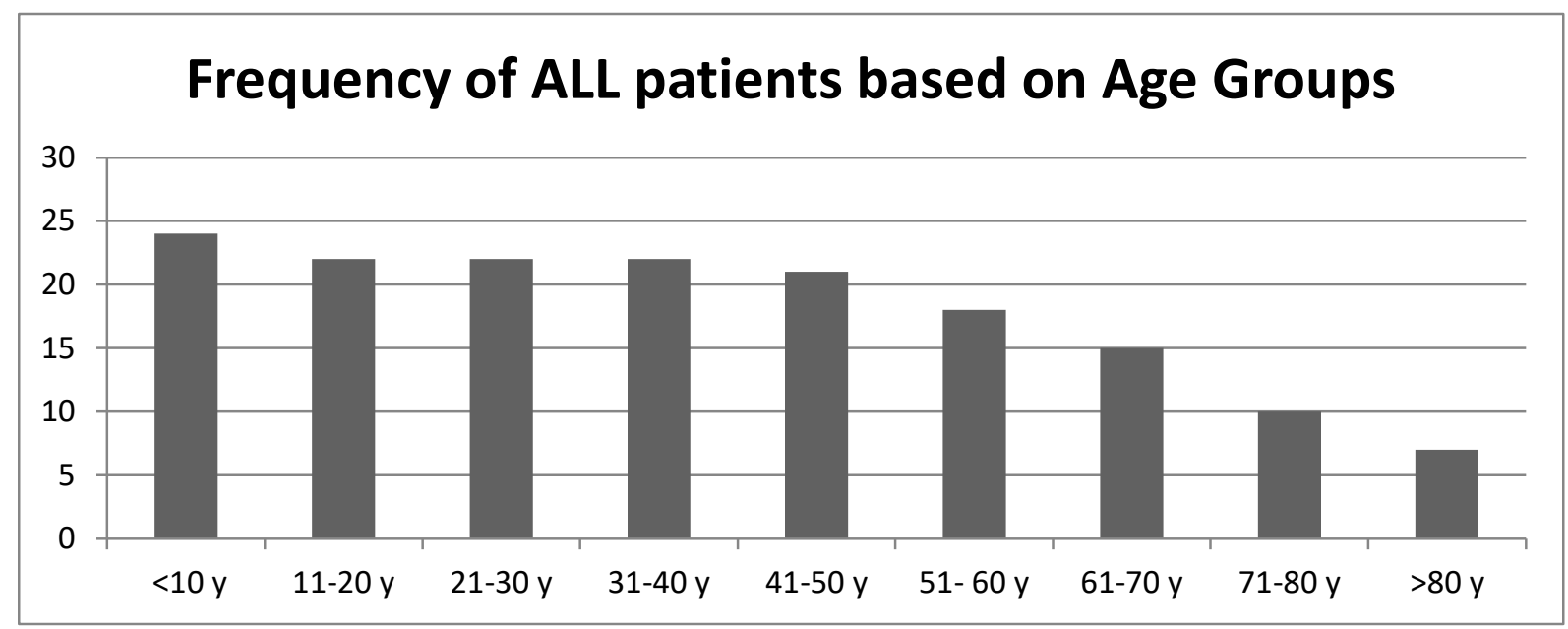

Figure 1. Frequency Distribution of ALL patient

Table 1. Percentage of patients based on age groupings

\begin{tabular}{|c|c|c|}
\hline S. No. & Age Groupings & Percentage \\
\hline 1 & $<10$ years & $24(14.90 \%)$ \\
\hline 2 & $10-20$ years & $22(13.66 \%)$ \\
\hline 3 & $21-30$ years & $22(13.66 \%)$ \\
\hline 4 & $31-40$ years & $22(13.66 \%)$ \\
\hline 5 & $41-50$ years & $21(13.04 \%)$ \\
\hline 6 & $51-60$ years & $18(11.18 \%)$ \\
\hline 7 & $61-70$ years & $15(09.31 \%)$ \\
\hline 8 & $71-80$ years & $7(06.21 \%)$ \\
\hline 9 & $>80$ years & $161(100 \%)$ \\
\hline & total & \\
\hline
\end{tabular}

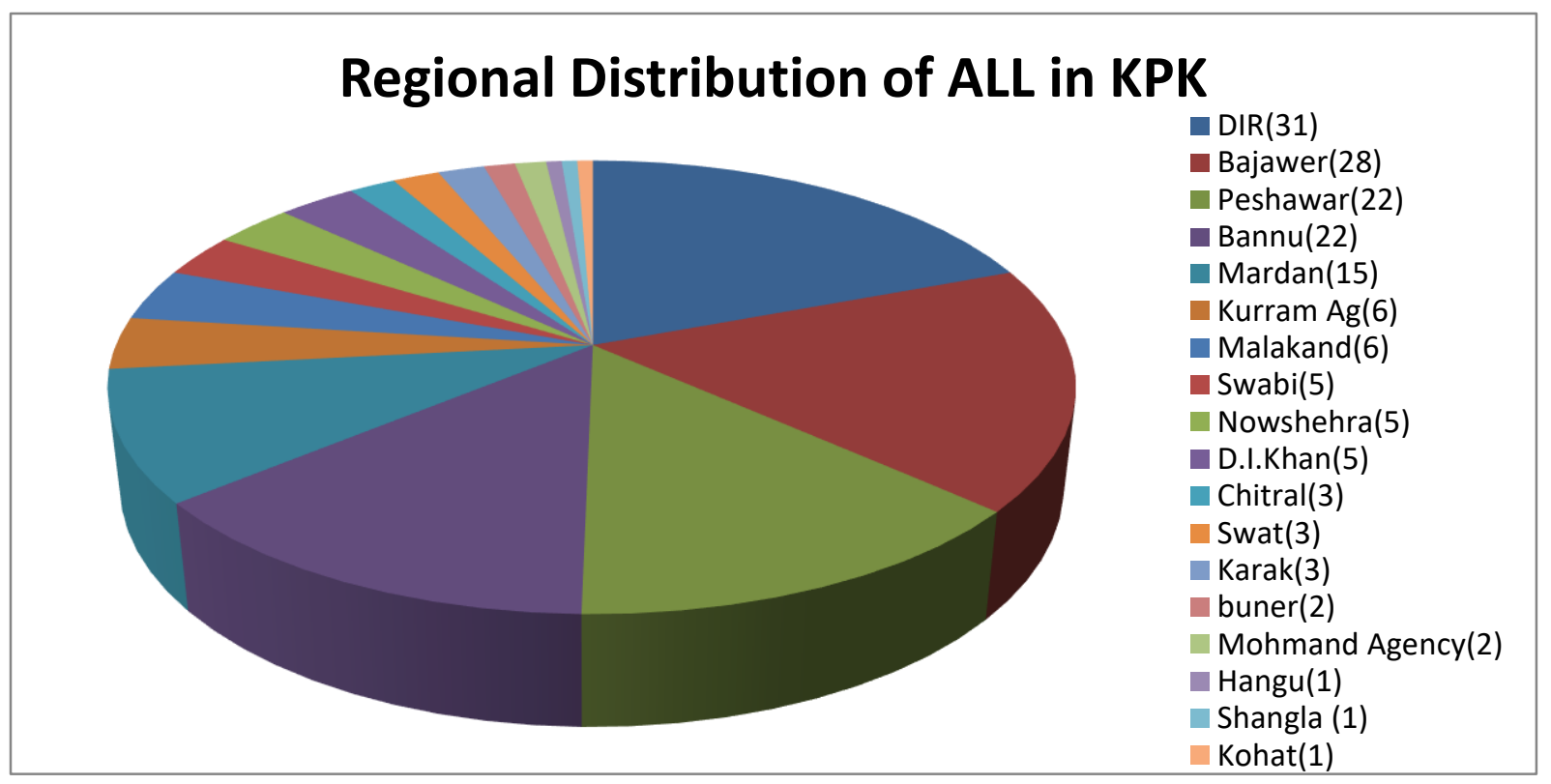

Figure 2. Region wise Distribution of ALL Patients 


\section{Discussion}

The current study found that acute lymphoblastic leukemia ALL) is slightly predominant in males. The cause of this gender disparity remains elusive. A study from the Hazara division [5] of KPK found the highest incidence rates of that leukemia compared to other cancers and also the disease was slightly more predominant in males [6]. According to the international agency on cancer research in Pakistan leukemia is ranked 5th based on incidence with a total number of cases $8305(4.9 \%)$ and the 5th cause of cancer-related deaths. A study from India [7] showed that ALL is slightly more common in males. All subtypes of ALL were more commonly reported in male gender vs females, T ALL 79.0\% males and $21.0 \%$ females, common ALL $55.6 \%$ vs $44.4 \%$ and similarly minor subtypes $62.5 \%$ male vs $37.5 \%$ in females. A study by Chandy [8] reported 6000 ALL cases in India with slight male predominance. The cause of such high cases in India is partly due to their large population size.

The mean age of ALL in our study was 34 years. This is in contrast to the literature where the mean age is usually under 10 years. The reason behind this age discrepancy is the strict criteria to enroll patients above 5 years of age and especially the adult population causing the bar to go upwards. Under 10 years are usually referred to other pediatric leukemic centers. An article in china by Chen et al. [9] similarly found ALL to be predominant in children with $70.28 \%$ of the total cases and overall common in males $60.33 \%$ vs females $39.67 \%$ with a median age of 10 years.ALL incidence was mainly in the pediatric age group especially under 10 years in the study. A similar study in India by Gurunet et al. [10] found ALL more prevalent in the pediatric age group with $76.4 \%$ under age 10 with a median age of presentation 6.5 years. A multi-center study conducted in the middle east [11] similarly found the mean age of ALL to be $6.1 \pm 3.9$ years and out of this sample size $59.2 \%$ were boys.

According to cancer research UK [12] found the highest incidence of ALL in age 0-4 years and then slowly decline and about 6\% ALL cases are in age $>75$ years, correlating with our study 0f $6.76 \%$ ALL cases over 70 years. American cancer registry data [13] found ALL peak in children less than 5 years of age and the risk then declines slowly until the mid-20s, and begins to rise again slowly after age 50. Our study found the highest incidence of ALL in the DIR \& Bajawer districts of KPK. The cause of its high incidence in these districts is unclear but it may be partly due to the leukemogenic chemicals which have soiled the earth during used in a war during the local unrest in 2008-2010. Also, the soil can have radiation particles in it leading to leukemic changes in the cells of the population being exposed. A study [14] found that the Tribal areas of KPK have higher values of ecological risk index, hazard quotient, and carcinogenic risk as compared to the other parts of Khyber Pakhtunkhwa. Another study [15] found that Cesium 137, a leukemogenic radioisotope being detected in the soil of KPK districts responsible for the higher incidence of ALL in KPK.

\section{Conclusion}

The present study evaluated the age, gender, and regional distribution of ALL in the KPK population. The study found that the incidence of acute lymphoblastic leukemia is slightly more common in males and under age 10 years which is in concordance with local, regional, and international studies. The study also highlights variations in the incidence of ALL in different regions of KPK, which can be attributed to leukemogenic chemicals and radioisotopes in the soil of different regions of KPK. This requires future studies in these regions for confirmation of ALL for any causal relationship. Moreover, strategies require to 
adopt for increasing public awareness and attitudes toward the risk factors and prevention of ALL.

\section{Authors' contributions}

Conceived and designed the experiments: Sajid A \& Abid J, Performed the experiments: Ayesha S \& Sufyan, Analyzed the data: Shehryar A, Shah H \& Sufyan, Contributed reagents/ materials/ analysis tools: Sajid A \& Ayesha S, Wrote the paper: Sajid A, Shehryar A \& Ayesha S.

\section{References}

1. Board PP (2021). Childhood Acute Lymphoblastic Leukemia Treatment (PDQ $\left.{ }^{\circledR}\right)$ : Patient Version. PDQ Cancer Information Summaries. October 7.

2. Puckett Y \& Chan O (2022). Acute Lymphocytic Leukemia. 2022 Jan 2. In: StatPearls [Internet]. Treasure Island (FL): Stat Pearls Publishing; Jan. PMID: 29083572.

3. Ismail M, Zia MA \& Khan HM (2017). Investigation of Cs-137 in the environmental soil segments of the Peshawar and Nowshera districts of Khyber Pakhtunkhwa, Pakistan. IJRR 15(4): 409-11.

4. Roberts KG (2018). Genetics and prognosis of ALL in children vs adults. Hematology Am Soc Hematol Educ Program. Nov 30; 1: 137-145.

5. Cancer today. International agency for research and cancer. https://gco.iarc.fr/today/ data /factsheets/populations/586-pakistanfact-sheets.

6. Ahmed S, Qureshi AN, Kazmi A, Rasool A, Gul M, Ashfaq M, Batool L, Rehman RA, Ahmad J \& Muniba (2013). First cancer statistics report from Hazara division. $J A y u b$ Med Coll Abbottabad 25(1-2): 71-73.

7. Kamat DM, Gopal R \& Advani SH (1985). Patterns of subtypes of Acute Lymphoblastic Leukemia in India. Leuk Res 9: 927-934.

8. Chandy M (1995). Childhood acute Iymphoblastic Ieukemia in India: an approach to management in a three-tier Society. Med Pediatr Oncol 25: 197-203.

9. Chen B, Wang YY, Shen Y, Zhang WN, He $\mathrm{HY}$, Zhu YM, Chen HM, Gu CH, Fan X, Chen JM, Cao Q, Yang G, Jiang CL, Weng XQ, Zhang XX, Xiong SM, Shen ZX, Jiang H, Gu LJ, Chen Z, Mi JQ \& Chen SJ (2012). Newly diagnosed acute lymphoblastic leukemia in China. INature.com/ Leukemia 26: $1608-1616$.

10. Guru FR, Muzamil J, Bashir S, Mahajan A (2018). Acute lymphoblastic leukemia, the Indian scenario. MOJ Cell Sci Rep 5(2): 3337.

11. Naima A. Al-Mulla, Chandra P, Khattab M, Madanat F, Vossough P, Torfa E, Al-Lamki Z \& Zain G (2014). Childhood acute lymphoblastic leukemia in the Middle East and neighboring countries: A prospective multi-institutional international collaborative study (CALLME1) by the Middle East Childhood Cancer Alliance (MECCA). Pedriatric Blood \& Cancer 61(8) 1403-1410.

12. https://www.cancerresearchuk.org/healthprofessional/cancer-statistics/statistics-bycancer-type/leukaemiaall/incidence\#heading-One.

13. American/cancer/society. Acute Lymphocytic Leukemia https://www.cancer.org/cancer/ acutelymphocytic-leukemia/about/keystatistics.html.

14. Saddique U, Muhammad S, Tariq M, Zhang H, Arif M, IA \& Khattak NU (2018). Potentially toxic elements in soil of the $\mathrm{K}$ Jadoon hyber Pakhtunkhwa province and Tribal areas, Pakistan: evaluation for human and ecological risk assessment. Pubmed 40(5): 2177-2190.

15. Ismail M, Zia MA \& Khan HM (2017). Investigation of Cs-137 in the environmental soil segments of the Peshawar and Nowshera districts of Khyber Pakhtunkhwa, Pakistan. Int J Rad Res 15(4): 407-412. 